\title{
Discretization Effects in the $\varepsilon$ Domain of QCD
}

\section{Mario Kieburg}

Fakultät für Physik, Postfach 100131, 33501 Bielefeld, Germany

E-mail: mkieburg@physik.uni-bielefeld.de

\section{K. Splittorff}

Discovery Center, The Niels Bohr Institute

University of Copenhagen,

Blegdamsvej 17, DK-2100 Copenhagen, Denmark

E-mail: splitenbi.dk

\section{Jacobus J. M. Verbaarschot*}

Department of Physics and Astronomy, State University of New York at Stony Brook, NY

11794-3800, USA

E-mail: jacobus.verbaarschotestonybrook.edu

\section{Savvas Zafeiropoulos}

Department of Physics and Astronomy, State University of New York at Stony Brook, NY

11794-3800, USA and

Laboratoire de Physique Corpusculaire, Université Blaise Pascal, CNRS/IN2P3 63177 Aubière

Cedex, France

E-mail: zafeiropouloseclermont.in2p3.fr

At nonzero lattice spacing the QCD partition function with Wilson quarks undergoes either a second order phase transition to the Aoki phase for decreasing quark mass or shows a first order jump when the quark mass changes sign. We discuss these phase transitions in terms of Wilson Dirac spectra and show that the first order scenario can only occur in the presence of dynamical quarks while in the quenched case we can only have a transition to the Aoki phase. The exact microscopic spectral density of the non-Hermitian Wilson Dirac operator with dynamical quarks is discussed as well. We conclude with some remarks on discretization effects for the overlap Dirac operator.

31st International Symposium on Lattice Field Theory - LATTICE 2013

July 29 - August 3, 2013

Mainz, Germany

\footnotetext{
*Speaker.
} 


\section{Introduction}

The Wilson term violates the anti-Hermiticity of the Dirac operator so that its eigenvalues will be scattered into the complex plane. When the quark mass is inside the strip of eigenvalues the partition function is in a phase known as the Aoki phase [1], and a reliable extrapolation to the continuum limit is not possible. This scenario happens in the quenched case for sufficiently small quark masses and temperature [2]. For dynamical quarks, a different scenario is possible. Because of the fermion determinant, the eigenvalues will be repelled from the quark mass and when the quark mass crosses zero a collective jump of the eigenvalues to the other side of the imaginary axis can take place [2]. This is known as the first order or Sharpe-Singleton scenario [3, 4], which was first discovered by means of Wilson chiral perturbation theory. In this scenario there is no transition to a different phase and extrapolation to the continuum limit is possible for all values of the quark mass, $m$, and of the lattice spacing, $a$. What happens can be analyzed by means of Wilson chiral perturbation theory. The terms in the chiral Lagrangian are determined by chiral symmetry in the same way as the terms involving the quark mass. The lowest order term in $a$ can be absorbed by a redefinition of the mass term leaving us with three terms of second order in $a$ with low-energy constants $W_{6 / 7 / 8}$. The spectrum of the Wilson Dirac operator can be obtained by extending the chiral Lagrangian with fermionic and bosonic valence quarks $[5,6,4,7]$. In the microscopic limit it can also be obtained by analyzing the equivalent random matrix theory (RMT) [7, 8, 9, 10]. Among others this provides us with simple relations to determine the low-energy constants [10]. One important effect of a non-zero lattice spacing is the broadening of the zero modes to a finite width $\sim a / \sqrt{V}$ (for small $a$ ) [7] as was first observed in [11]. Other analytical predictions for the Wilson Dirac spectra have also been confirmed by lattice simulations [12, 13, 14].

Because of the absence of an exact chiral symmetry it is hard to approach the chiral limit with Wilson fermions, but this is possible by means of the overlap Dirac operator [15] or by domain wall fermions [16]. Since the overlap Dirac operator is based on the Wilson Dirac operator, we expect that it will inherit a remnant of the discretization effects. In particular, if the Wilson Dirac operator is in the Aoki phase, one may question if the overlap operator will not describe the continuum limit of lattice QCD. We investigate this in the framework of a random matrix model for the Wilson Dirac operator discussed in Sec. 2. Moreover we discuss the relation between the spectra of the Wilson Dirac operator and the overlap Dirac operator in Sec. 3.

\section{Wilson Chiral Perturbation Theory}

The Wilson Dirac operator has the following block structure

$$
D_{\mathrm{W}}=\left(\begin{array}{cc}
a A & i W \\
i W^{\dagger} & a B
\end{array}\right)
$$

with $W$ a complex matrix and $A$ and $B$ Hermitian matrices. This operator is $\gamma_{5}$-Hermitian $\left(D_{\mathrm{W}}^{\dagger}=\right.$ $\left.\gamma_{5} D_{\mathrm{W}} \gamma_{5}\right)$ so that its eigenvalues occur in complex conjugate pairs or are real. In the $\varepsilon$ domain of QCD $\left(V \rightarrow \infty\right.$ while $m V$ and $a^{2} V$ are kept constant $)$ the chiral Lagrangian is given by $[3,17,18,7]$.

$$
\begin{aligned}
& -\mathscr{L}=\frac{1}{2} m V \Sigma \operatorname{Tr}\left(U+U^{\dagger}\right)-\frac{1}{2} z V \Sigma \operatorname{Tr}\left(U-U^{\dagger}\right) \\
& -a^{2} V W_{6}\left[\operatorname{Tr}\left(U+U^{\dagger}\right)\right]^{2}-a^{2} V W_{7}\left[\operatorname{Tr}\left(U-U^{\dagger}\right)\right]^{2}-a^{2} V W_{8} \operatorname{Tr}\left(U^{2}+U^{-2}\right) .
\end{aligned}
$$


The trace squared $O\left(a^{2}\right)$ terms can be linearized by introducing a random mass [19]. For $W_{6}<0$ we have

$$
\exp \left[-a^{2} V W_{6} \operatorname{Tr}^{2}\left(U+U^{-1}\right)\right]=\int_{-\infty}^{\infty} \frac{d y}{\sqrt{16 \pi V\left|W_{6}\right| a^{2}}} \exp \left[-\frac{y^{2}}{16 V\left|W_{6}\right| a^{2}}-\frac{y}{2} \operatorname{Tr}\left(U+U^{-1}\right)\right],
$$

and the term proportional to $W_{7}$ can be linearized as (for $W_{7}<0$ )

$$
\exp \left[-a^{2} V W_{7} \operatorname{Tr}^{2}\left(U-U^{-1}\right)\right]=\int_{-\infty}^{\infty} \frac{d y}{\sqrt{16 \pi V\left|W_{7}\right| a^{2}}} \exp \left[-\frac{y^{2}}{16 V\left|W_{7}\right| a^{2}}-\frac{y}{2} \operatorname{Tr}\left(U-U^{-1}\right)\right] \text {. }
$$

Therefore, the trace squared terms can be generated by a random mass for the real part of the eigenvalues of $D_{\mathrm{W}}$ (for $W_{6}$ ) and a chiral random mass for the eigenvalues of $D_{5}=\gamma_{5} D_{\mathrm{W}}$ (for $W_{7}$ ). For the opposite sign of $W_{6}$ and $W_{7}$, these terms cannot originate from eigenvalue fluctuations [2]. The sign of $W_{8}$ follows from positivity requirements of the partition function [19] at fixed index $v$ of the Dirac operator yielding

$$
W_{8}-W_{6}-W_{7}>0 \text {. }
$$

Another inequality follows from the width of the strip parallel to the imaginary axis forming the support of the eigenvalues of $D_{\mathrm{W}}$ which for small $a$ is proportional to $W_{8}-2 W_{6}$ so that

$$
W_{8}-2 W_{6}>0
$$

Using partially quenched chiral perturbation theory, it can additionally be shown that $W_{8}>0$ independently of the value of $W_{6}$ and $W_{7}$ [20]. In Table 1 we give some recent results [21] for the low energy constants which are consistent with the inequalities given above.

\begin{tabular}{c|cc} 
& $W_{6}^{\prime}$ & $W_{8}^{\prime}$ \\
\hline Iwasaki & $0.0049(38)$ & $-0.0119(17)$ \\
tlSym & $0.0082(34)$ & $-0.0138(22)$
\end{tabular}

Table 1: Recent lattice results [21] for the low energy constants $W_{6}^{\prime}=-W_{6}$ and $W_{8}^{\prime}=-W_{8}$.

It is instructive to interpret the first order scenario in terms of eigenvalues of the Dirac operator. Because of the random mass, due to $W_{6}$, the strip of eigenvalues exhibits collective fluctuations. For dynamical quarks, this strip is repelled from the quark mass. However, when the quark mass crosses zero, the strip moves to the other side of the imaginary axis. A first order transition takes place if its half-width is less than the position of its center. The half-width of the strip is equal to $8 W_{8} a^{2} / \Sigma$. The distribution of the Gaussian random mass is modified by the two flavor partition function (which is equal to $\exp (2 \Sigma V|m-y|)$ in the mean field limit) and is thus given by [2]

$$
\exp \left[-\frac{y^{2} \Sigma^{2} V}{16\left|W_{6}\right| a^{2}}\right] \rightarrow \exp \left[-\frac{y^{2} \Sigma^{2} V}{16\left|W_{6}\right| a^{2}}+2 \Sigma V|m-y|\right],
$$

which is a Gaussian centered at $\pm 16\left|W_{6}\right| a^{2} / \Sigma$. We thus find a first order scenario if $8 W_{8} a^{2} / \Sigma<$ $16\left|W_{6}\right| a^{2} / \Sigma$ and, because of $W_{6}<0$, we have the condition $W_{8}+2 W_{6}<0$ for the first order scenario to occur [4]. The role of the fermion determinant is essential in this argument. Without it, the distribution of the random mass will be a Gaussian centered at zero so that a first order scenario cannot occur. Indeed, in the quenched case only a transition to the Aoki phase has been observed in lattice simulations see [2, 22]. 

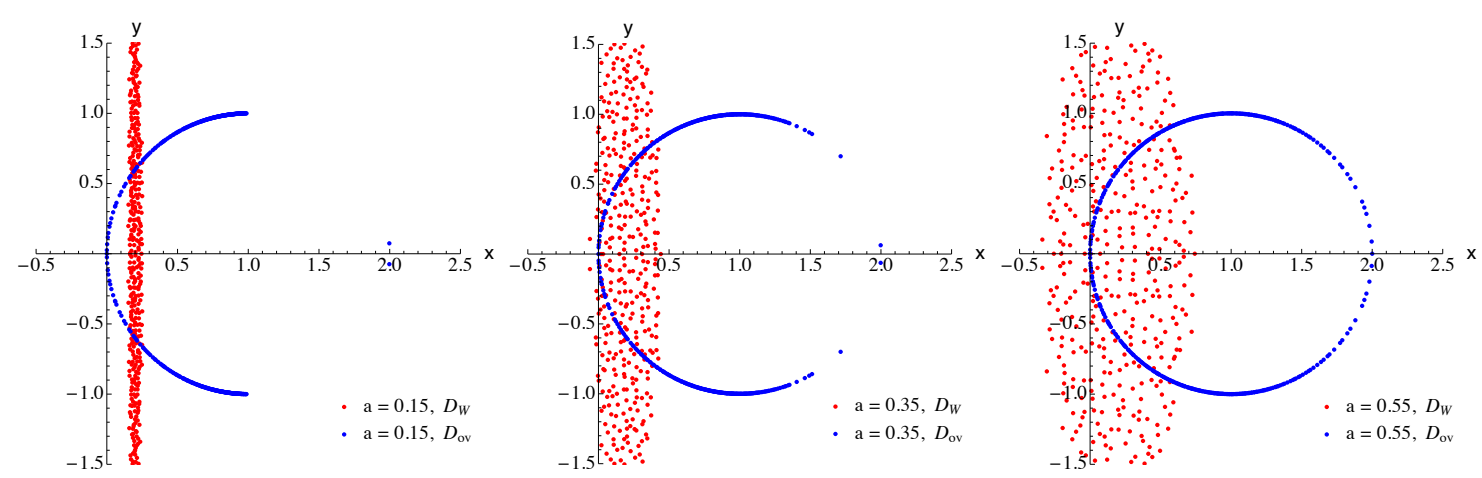

Figure 1: Scatter plot of the eigenvalues of a single Wilson Dirac operator (red) and its corresponding overlap Dirac operator (blue) with $m=0.2$ and different $a$ drawn from a random matrix ensemble with index $v=1$ and a $200 \times 201$ off-diagonal block $W$.

\section{The Overlap Dirac Operator}

In this section, we study the effect of the Wilson term on spectra of the overlap Dirac operator using the random matrix Wilson Dirac operator sharing the block structure of the Wilson Dirac operator (2.1) whose matrix elements are replaced by independent Gaussian random numbers. This RMT for the Wilson Dirac operator yields the equivalent microscopic Lagrangian (2.2). The corresponding massless overlap Dirac operator is defined in the usual way, [15, 23, 24, 25, 26, 27]

$$
D_{\mathrm{ov}}=1-\gamma_{5} U \operatorname{sign}\left(\Lambda_{5}\right) U^{-1}, \quad D_{5}=\left(D_{\mathrm{W}}+m\right) \gamma_{5}=U \Lambda_{5} U^{-1},
$$

where $U$ is the unitary matrix that diagonalizes $D_{5}$ to its eigenvalues $\Lambda_{5}$.

It looks drastic to replace the eigenvalues by their sign, but at vanishing lattice spacing this does not change the theory. The reason is that eigenvectors contain the information on the eigenvalues. For $a=0$, the Dirac operator $D_{5}$ can be block-diagonalized as

$$
D_{5}=\left(\begin{array}{ll}
u & 0 \\
0 & v
\end{array}\right)\left(\begin{array}{cc}
m & \lambda_{k} \\
\lambda_{k} & -m
\end{array}\right)\left(\begin{array}{cc}
u^{-1} & 0 \\
0 & v^{-1}
\end{array}\right)
$$

with $u$ and $v$ unitary. Performing an additional rotation by $\tan \left(2 \phi_{k}\right)=\lambda_{k} / m$ results in

$$
\left(\begin{array}{cc}
m & \lambda_{k} \\
\lambda_{k} & -m
\end{array}\right)=\left(\begin{array}{cc}
\cos \phi_{k} & -\sin \phi_{k} \\
\sin \phi_{k} & \cos \phi_{k}
\end{array}\right)\left(\begin{array}{cc}
\sqrt{\lambda_{k}^{2}+m^{2}} & 0 \\
0 & -\sqrt{\lambda_{k}^{2}+m^{2}}
\end{array}\right)\left(\begin{array}{cc}
\cos \phi_{k} & \sin \phi_{k} \\
-\sin \phi_{k} & \cos \phi_{k}
\end{array}\right) .
$$

After projection onto the sign of the eigenvalues, this rotation reproduces the left-hand of Eq. (3.3) rescaled by a factor $1 / \sqrt{\lambda_{k}^{2}+m^{2}}$ so that the eigenvalues of the overlap Dirac operator are given by

$$
\lambda_{\mathrm{ov}, \mathrm{k}}=1-\frac{m \pm i \lambda_{k}}{\sqrt{\lambda_{k}^{2}+m^{2}}}=1-e^{ \pm 2 i \phi_{k}} .
$$

The stereographic projection of the eigenvalues onto the imaginary axis is given by

$$
\lambda_{\mathrm{p}, \mathrm{k}}=\frac{\lambda_{\mathrm{ov}, \mathrm{k}}}{1-\lambda_{\mathrm{ov}, \mathrm{k}} / 2}=\mp 2 i \tan \phi_{k}=\mp 2 i \frac{\lambda_{k}}{m+\sqrt{\lambda_{k}^{2}+m^{2}}},
$$



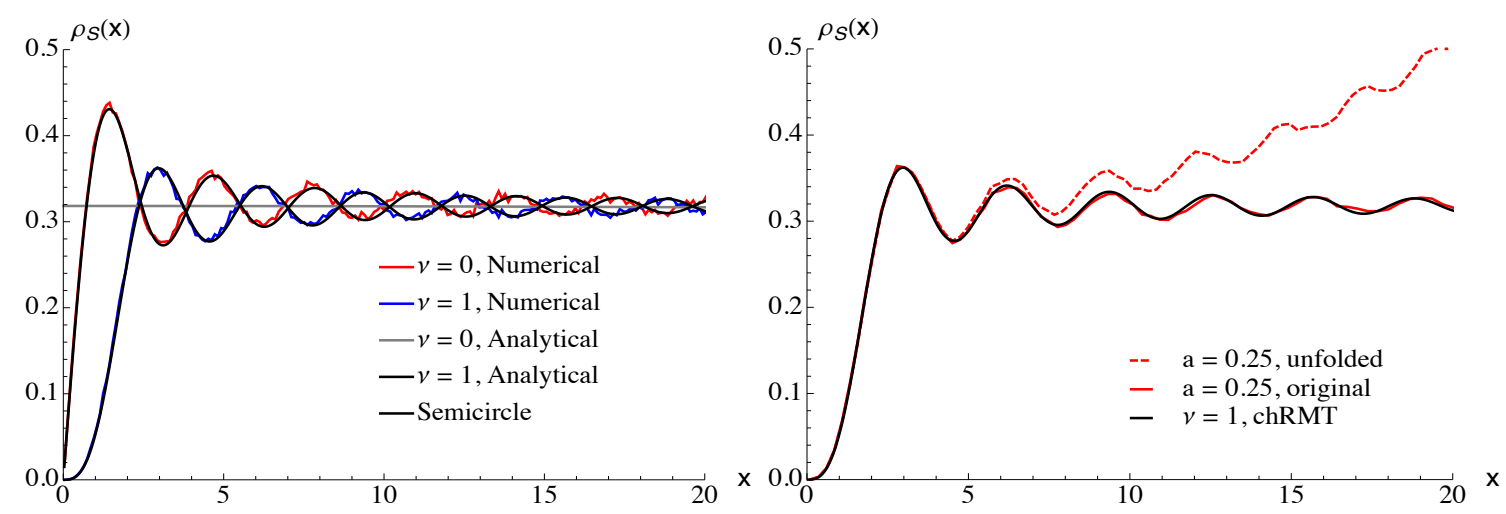

Figure 2: Left: The spectral density of the projected overlap Dirac operator obtained from a Wilson kernel with a large mass $m=100, a=0.3$ and index $v=0$ and $v=1$. The black curve shows the analytical continuum result and the red and blue curve show the result from the computed eigenvalues. The grey curve is the mean field result for the spectral density which is a semicircle with height $1 / \pi$ at the origin. Right: Comparison of the spectral density of the overlap Dirac operator for $a=0.15$ and $m=0.2$ (Red) before (dashed) and after unfolding (full) and the analytical continuum chiral RMT result (black) [29].

which for large mass, $m$, simplifies to

$$
\lambda_{\mathrm{p}, \mathrm{k}} \approx \mp i \frac{\lambda_{k}}{m}+O\left(\frac{\lambda_{k}^{2}}{m^{2}}\right) .
$$

At nonzero lattice spacing the overlap operator preserves a new form of chiral symmetry [28] so that the expected pattern of chiral symmetry breaking and eigenvalue correlations are the same as in the continuum limit. In Fig. 1 we show scatter plots of eigenvalues of the random matrix Wilson Dirac operator and the corresponding overlap Dirac operator. For large Wilson mass, the eigenvalues move close to zero, and after rescaling we indeed find close agreement with the continuum chRMT result (see Fig. 2).

For smaller Wilson mass or larger lattice spacing the eigenvalues of the overlap Dirac operator cover an increasing part of the unit circle, and in the Aoki phase, the spectral density becomes nonvanishing everywhere on the unit circle. In the normal phase the real eigenvalues of the overlap operator are necessarily located at zero while in the Aoki phase they can be either at $\lambda=0$ or $\lambda=2$ (see Fig. 1). As a consequence, the number of zero modes which determines the microscopic spectral density is not equal to the index of the Wilson kernel and the spectral density is a superposition of the spectra with a different number of zero modes. This redistribution of the index of the Wilson kernel may affect the $\theta$-dependence of the overlap partition function which will be studied elsewhere.

The spectral behavior of $D_{\mathrm{ov}}$ is illustrated by simulations of the overlap operator with the Wilson RMT kernel, see Eq. (2.1). In Fig. 3 spectra of $D_{\mathrm{ov}}$ are shown for various lattice spacings and a quark mass of $m=0.2$ with the index of the Wilson kernel equal to $v=1$. After rescaling the eigenvalues, the curves around the origin collapse onto a single curve outside the Aoki phase and to a different curve inside this phase (see Fig. 3). The reason is that, in the Aoki phase, the ensemble is a superposition of configurations with 0 or 1 zero modes with a very small admixture of more zero modes. Hence, the spectral density is a superposition of $v=0$ and $v=1$ Dirac spectra with each of them given by the continuum chRMT result. 

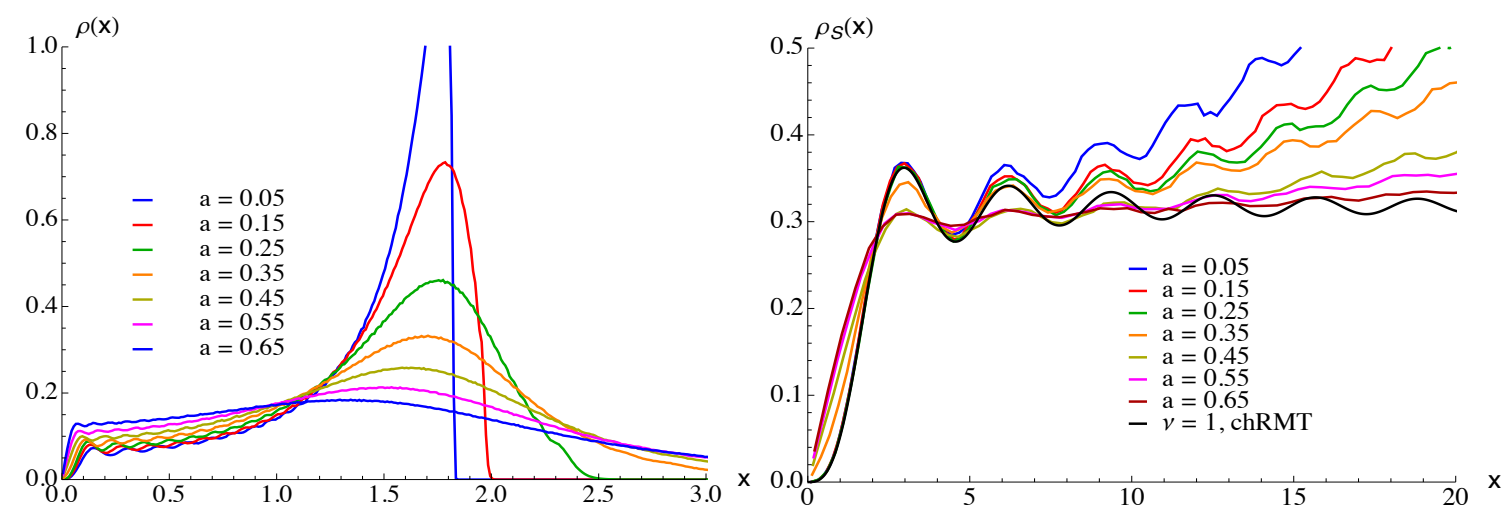

Figure 3: The spectral density of the projected overlap Dirac spectra for Wilson kernel with index $v=1$ and a small kernel mass $m=0.2$ and $a=0.05, a=0.15, a=0.25, a=0.35, a=0.45, a=0.55, a=0.65$ (from top to bottom). The critical value for the transition to the Aoki phase is $a=0.35$ (green curve). In the right figure the curves have been rescaled, but otherwise the data are the same.

The agreement of the spectral density with continuum chiral RMT (chRMT) for the values of $a$ outside the Aoki phase can better be seen after unfolding the spectrum, see Fig. 2. Thereby, the spectrum is transformed to have a constant average level spacing between consecutive levels.

\section{Conclusions}

Wilson chiral perturbation theory and the corresponding Wilson RMT are powerful tools to study discretization effects of the Wilson Dirac operator. In the microscopic domain as well as in the mean field limit this allows us to derive exact results for the spectra of the Wilson Dirac operator and the Hermitian Wilson Dirac operator. Among others, the topological eigenvalues of the Dirac operator are broadened with a width that for small $a$ scales as $a / \sqrt{V}$. The width of the spectrum $D_{\mathrm{W}}$ is directly related to the low-energy constants. For small lattice spacing we have derived simple relations between properties of Dirac spectra and low energy constants. In this lecture, we have discussed the mean field result for the Wilson Dirac spectrum with dynamical quarks and have argued that the Dirac spectrum shows a first order jump for $W_{8}+2 W_{6}<0$. This jump is induced by the fermion determinant and only occurs in the presence of dynamical quarks.

Since lattice theories with the overlap Dirac operator preserve a form of chiral symmetry at nonzero lattice spacing we expect that the eigenvalues behave as in the continuum. Indeed, after unfolding, the correlations of the overlap Dirac operator are given by continuum chRMT. This is robust as long as the Wilson Dirac operator in the kernel is outside the Aoki phase. Inside the Aoki phase, we find a superposition of chRMT spectra determined by the distribution of the zero modes.

Acknowledgments. This work was supported by U.S. DOE Grant No. DE-FAG-88FR40388 (JV and SV), the Humboldt Foundation (MK) and the Sapere Aude program of The Danish Council for Independent Research (KS). We thank Gernot Akemann and Poul Damgaard for fruitful discussions. 


\section{References}

[1] S. Aoki, Phys. Rev. D 30 (1984) 2653.

[2] M. Kieburg, K. Splittorff and J. J. M. Verbaarschot, Phys. Rev. D 85, 094011 (2012).

[3] S. R. Sharpe and R. L. Singleton, Phys. Rev. D 58, 074501 (1998).

[4] M. Golterman, S. R. Sharpe, R. L. Singleton, Jr., Phys. Rev. D71, 094503 (2005).

[5] S. R. Sharpe, Phys. Rev. D 74, 014512 (2006).

[6] S. Necco, A. Shindler, JHEP 1104, 031 (2011).

[7] P. H. Damgaard, K. Splittorff and J. J. M. Verbaarschot, Phys. Rev. Lett. 105, 162002 (2010).

[8] M. Kieburg, J. J. M. Verbaarschot and S. Zafeiropoulos, Phys. Rev. Lett. 108, 022001 (2012).

[9] G. Akemann, T. Nagao, JHEP 1110, 060 (2011).

[10] M. Kieburg, J. J. M. Verbaarschot and S. Zafeiropoulos, Phys. Rev. D 88, 094502 (2013).

[11] L. Del Debbio, L. Giusti, M. Lüscher, R. Petronzio and N. Tantalo, JHEP 0602, 011 (2006); JHEP 0702, 056 (2007).

[12] A. Deuzeman, U. Wenger and J. Wuilloud, JHEP 1112, 109 (2011).

[13] P. H. Damgaard, U. M. Heller and K. Splittorff, Phys. Rev. D 85, 014505 (2012).

[14] P. H. Damgaard, U. M. Heller and K. Splittorff, Phys. Rev. D 86, 094502 (2012).

[15] H. Neuberger, Phys. Lett. B 417, 141 (1998).

[16] D. B. Kaplan, Phys. Lett. B 288, 342 (1992).

[17] G. Rupak and N. Shoresh, Phys. Rev. 66, 054503 (2002).

[18] O. Bär, G. Rupak and N. Shoresh, Phys. Rev. D 70, 034508 (2004).

[19] G. Akemann, P. H. Damgaard, K. Splittorff, J. J. M. Verbaarschot, Phys. Rev. D 83, 085014 (2011).

[20] M. T. Hansen and S. R. Sharpe, Phys. Rev. D 85, 014503 (2012).

[21] G. Herdoiza, K. Jansen, C. Michael, K. Ottnad and C. Urbach, JHEP 1305, 038 (2013).

[22] K. Splittorff, PoS LATTICE 2012, 018 (2012).

[23] R. Narayanan and H. Neuberger, Nucl. Phys. B 412, 574 (1994).

[24] R. Narayanan and H. Neuberger, Phys. Rev. Lett. 71, 3251 (1993).

[25] R. Narayanan and H. Neuberger, Nucl. Phys. B 443, 305 (1995).

[26] R. G. Edwards, U. M. Heller and R. Narayanan, Phys. Rev. D 59, 094510 (1999).

[27] K. Splittorff and A. D. Jackson, [hep-lat/9805018].

[28] M. Lüscher, Phys. Lett. B 428, 342 (1998).

[29] E. V. Shuryak and J. J. M. Verbaarschot, Nucl. Phys. A 560, 306 (1993); J. J. M. Verbaarschot and I. Zahed, Phys. Rev. Lett. 70, 3852 (1993); J. J. M. Verbaarschot, Phys. Rev. Lett. 72, 2531 (1994). 